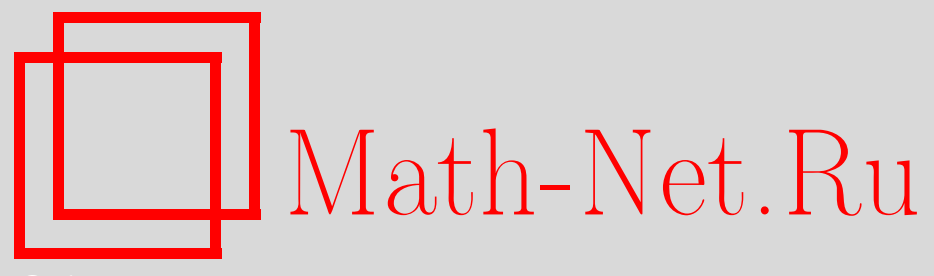

И. Н. Шнурников, О числе областей максимальной размерности в разбиениях проективных пространств наборами гиперплоскостей, Матем. заметки, 2015, том 97, выпуск 3, 475-480

DOI: https://doi.org/10.4213/mzm10602

Использование Общероссийского математического портала Math-Net.Ru подразумевает, что вы прочитали и согласны с пользовательским соглашением http://www . mathnet.ru/rus/agreement

Параметры загрузки:

IP: 54.224 .135 .184

26 апреля 2023 г., 12:26:15

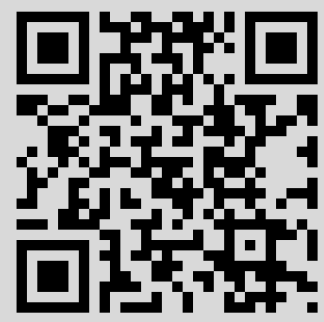




\section{О числе областей максимальной размерности в разбиениях проективных пространств наборами гиперплоскостей}

\section{И. Н. Шнурников}

Введение. Грюнбаум в [1] систематично изучал комбинаторные характеристики наборов $n$ прямых на проективной плоскости: возможные значения и соотношения между числами $v, e, f, t_{i}$ и $p_{j}$ точек пересечения, отрезков прямых, областей, точек пересечения кратности $i$ и $j$-угольных областей соответственно. В частности, частично описал множество возможных значений числа $f$ связных компонент (областей) дополнения к объединению прямых (не обязательно общего положения). Мартинов в [2] полностью описал множества чисел $f$ для наборов прямых и псевдопрямых. Шеннон в [3] получил нижние точные оценки для числа $k$-мерных плоскостей и для числа $k$-мерных клеток в разбиениях вещественного проективного пространства $\mathbb{R P}^{d}$ нетривиальными наборами из $n$ гиперплоскостей и нашел наборы, на которых оценки достигаются. Заславский в [4] выразил число областей дополнения к наборам гиперплоскостей в терминах комплекса пересечений гиперплоскостей. Бак в [5] нашел максимальные возможные значения числа $k$-мерных клеток в разбиениях пространства $\mathbb{R} \mathbb{P}^{d}$ наборами $n$ гиперплоскостей.

Настоящая работа является продолжением и обобщением упомянутых результатов Грюнбаума, Шеннона и Мартинова; используются результаты Заславского и свойства геометрических решеток. Приложения работы могут быть ввиду наличия связей между комбинаторикой наборов $\mathscr{A}$ вещественных гиперплоскостей и топологией дополнений $\mathbb{C}^{n} \backslash \mathscr{A}_{c}$ к комплексифицированным гиперплоскостям; например, число областей $f$ в вещественной конфигурации совпадает с размерностью кольца когомологий дополнения $\mathbb{C}^{n} \backslash \mathscr{A}_{c}$, см. работу Орлика и Соломон [6]. Дешпанд в [7] обобщил характеристический многочлен на конфигурации подмногообразий, ввиду чего представляемая работа может быть обобщена на конфигурации подмногообразий, с соответствующими неравенствами (оценками). Есть также результаты многих других авторов о конфигурациях (гипер)плоскостей, которые здесь не упомянуты потому, что непосредственно не используются. Возможна связь работы с оценкой числа пороговых функций двузначной логики. Число пороговых функций (булевых $n$-местных функций, задаваемых условием $\sum a_{i} x_{i} \geqslant c$ ) совпадает с числом областей в конкретной конфигурации из $2^{n}$ гиперплоскостей $\pm a_{1} \pm a_{2} \cdots \pm a_{n}=c$. Тем самым, полученные в работе нижние оценки числа областей в вырожденных конфигурациях гиперплоскостей могут быть использованы для улучшения нижних оценок числа пороговых функций.

Постановка задачи: рассматривается объединение $n$ различных гиперплоскостей размерности $d-1$ в вещественном проективном $d$-мерном пространстве $\mathbb{R} \mathbb{P}^{d}$, причем пересечение всех гиперплоскостей - пустое множество. Описать множество $F_{d}(n)$ всех возможных значений числа $f$ связных компонент дополнения в $\mathbb{R} \mathbb{P}^{d}$ к объединению $n$ гиперплоскостей. Упорядочив элементы множества $F_{d}(n)$ по возрастанию, найти несколько первых элементов и доказать, что целые числа между ними не реализуются в качестве числа областей.

Основной результат данной работы - следующая теорема.

Теорема 1. Пусть $d \geqslant 3 u n \geqslant 2 d+5$. Тогда первые четыре элемента множества $F_{d}(n)$, упорядоченного по возрастанию, следующие:

$$
(n-d+1) 2^{d-1}, \quad 3(n-d) 2^{d-2}, \quad(3 n-3 d+1) 2^{d-2}, \quad 7(n-d) 2^{d-3} .
$$

В отличии от работ Шеннона, ищется не (одно) наименьшее число областей, а несколько первых по возрастанию реализуемых чисел областей (при этом первое из них, естественно,

DOI: $10.4213 / \mathrm{mzm} 10602$ 
совпадает с числом Шеннона). В отличии от работ Мартинова [2] и автора [8], рассматриваются наборы гиперплоскостей в пространстве $\mathbb{R} \mathbb{P}^{d}$ при $d \geqslant 3$. При этом первые три числа множества $F_{d}(n)$ из теоремы 1 данной работы в случае размерности $d=3$ не превосходят $6 n-16$ и совпадают с числами, найденными в [8].

Определения и вспомогательные утверждения. Проективные подпространства размерностей $i$ и $j$ пространства $\mathbb{R P}^{d}$ находятся в общем положении, если их пересечение есть $(i+j-d)$-мерное проективное подпространство при $i+j \geqslant d$ и пустое множество при $i+j<d$. Скажем, что проективное подпространство находится в общем положении относительно конфигурации гиперплоскостей, если оно находится в общем положении с гиперплоскостями и всеми непустыми пересечениями любого количества гиперплоскостей. Тривиальными называются наборы, все гиперплоскости в которых имеют общую точку. Минимальными ${ }^{1}$ называются (нетривиальные) наборы $n$ гиперплоскостей в $\mathbb{R P}^{d}$, в которых $n-d+1$ гиперплоскостей имеют общую $(d-2)$-мерную плоскость, а остальные $d-1$ гиперплоскостей находятся в общем положении (каждая по отношению ко всем остальным). Для набора $\mathscr{A}$, состоящего из $n$ гиперплоскостей размерности $d-1$ в пространстве $\mathbb{R} \mathbb{P}^{d}$, через $f=f(\mathscr{A})$ обозначим число связных компонент дополнения в пространстве $\mathbb{R} \mathbb{P}^{d}$ к объединению гиперплоскостей набора; компоненты связности будем называть областями. Множество всех чисел $f$ для нетривиальных наборов гиперплоскостей обозначается через $F_{d}(n)$. Наибольший элемент множества $F_{d}(n)$ равен $1+C_{n-1}^{1}+\cdots+C_{n-1}^{d}$ при $d \leqslant n-1$ и достигается для наборов гиперплоскостей в общем положении, см. [5]. Для набора $\mathscr{A}$ гиперплоскостей в пространстве $\mathbb{R P}^{d}$ через $m=m(\mathscr{A})$ обозначим максимальное число гиперплоскостей, имеющих общую точку.

Лемма 1 (Шеннон, [3]). Наименьший элемент множества $F_{d}(n)$ равен $(n-d+1) 2^{d-1}$, причем реализуется только на минимальных наборах.

Пусть $p$ - одна из гиперплоскостей набора $\mathscr{A}$. Через $\mathscr{A}^{\prime}$ обозначим набор остальных гиперплоскостей из набора $\mathscr{A}$, отличных от $p$. Через $\mathscr{A}^{\prime \prime}$ обозначим набор пересечений гиперплоскости $p$ с гиперплоскостями из набора $\mathscr{A}^{\prime}$, т.е. $\mathscr{A}^{\prime \prime}$ - это набор $(d-2)$-мерных плоскостей в $(d-1)$-мерной плоскости $p$. Тройкой наборов назовем тройку $\left(\mathscr{A}, \mathscr{A}^{\prime}, \mathscr{A}^{\prime \prime}\right)$. Через $L(\mathscr{A})$ обозначим упорядоченное обратно включению множество всех непустых пересечений гиперплоскостей из набора, включая все проективное пространство. Элементы множества $L(\mathscr{A})$ будем называть плоскостями.

ОПределЕниЕ 1. Функиией Мёбиуса $\mu: L(\mathscr{A}) \times L(\mathscr{A}) \rightarrow \mathbb{Z}$ называется рекуррентно заданная функция

$$
\mu(u, v)= \begin{cases}0, & \text { если } u \npreceq v ; \\ 1, & \text { если } u=v ; \\ -\sum_{u \preceq w \prec v} \mu(u, w), & \text { если } u \prec v .\end{cases}
$$

Если первый аргумент функции Мебиуса - это объемлющее пространство $V$, то его будем опускать, т.е. писать $\mu(w)$ вместо $\mu(V, w)$ для плоскости $w$. Для множеств пересечений наборов $\mathscr{A}, \mathscr{A}^{\prime}$ и $\mathscr{A}^{\prime \prime}$ соответствующие функции Мёбиуса обозначим через $\mu, \mu^{\prime}$ и $\mu^{\prime \prime}$.

ЛЕмма 2 (Заславский, [4]). Для тройки наборов $\left(\mathscr{A}, \mathscr{A}^{\prime}, \mathscr{A}^{\prime \prime}\right)$ верно

$$
f(\mathscr{A})=f\left(\mathscr{A}^{\prime}\right)+f\left(\mathscr{A}^{\prime \prime}\right) .
$$

Лемма 3. Пусть $\left(\mathscr{A}, \mathscr{A}^{\prime}, \mathscr{A}^{\prime \prime}\right)$ - тройка наборов, плоскость $v \in L(\mathscr{A})$. Тогда

$$
\mu(v)= \begin{cases}\mu^{\prime}(v)-\mu^{\prime \prime}(v), & \text { если } v \in L\left(\mathscr{A}^{\prime}\right), v \in L\left(\mathscr{A}^{\prime \prime}\right), \\ -\mu^{\prime \prime}(v) & \text { еслu } v \notin L\left(\mathscr{A}^{\prime}\right), v \in L\left(\mathscr{A}^{\prime \prime}\right), \\ \mu^{\prime}(v) & \text { еслu } v \in L\left(\mathscr{A}^{\prime}\right), v \notin L\left(\mathscr{A}^{\prime \prime}\right) .\end{cases}
$$

\footnotetext{
${ }^{1}$ Перевод термина near pencil.
} 
ДоказАтельство. Следует из определения функции Мёбиуса индукцией по коразмерности плоскости $v$, начиная с 0.

Лемма 4 (Заславский, [4]). Для набора $\mathscr{A}$ гиперплоскостей в проективном пространcmвe $\mathbb{R} \mathbb{P}^{d}$

$$
f(\mathscr{A})=\sum_{v \in L(\mathscr{A}), 2 \mid \operatorname{dim}(v)}|\mu(v)| .
$$

Лемма 5. Для нетривиальных наборов гиперплоскостей

$$
f \geqslant(n-m+1)(m-d+2) 2^{d-2} .
$$

ДоказАтельство. Обозначим через $p_{1}, \ldots, p_{m}$ какие-нибудь $m$ гиперплоскостей с непустым пересечением $Q$. Размерность $Q$ равна нулю, а гиперплоскости $p_{1}, \ldots, p_{m}$ получаются взятием конуса с центром в $Q$ над некоторым набором $\mathscr{B}$ из $m$ гиперплоскостей в $(d-1)$-мерном подпространстве. По лемме 1 имеем $f_{d-1}(\mathscr{B}) \geqslant(m-d+2) 2^{d-2}$. На каждой из остальных гиперплоскостей набора плоскости $p_{1}, \ldots, p_{m}$ высекают конфигурацию, проективно эквивалентную $\mathscr{B}$. Поэтому при каждом добавлении к гиперплоскостям $p_{1}, \ldots, p_{m}$ остальных $n-m$ гиперплоскостей одна за одной число областей будет увеличиваться не менее, чем на $(m-d+2) 2^{d-2}$.

Лемма 6. Если плоскость $v \in L(\mathscr{A})$ принадлежит $j$ гиперплоскостям набора, то

$$
|\mu(v)| \geqslant j-d+\operatorname{dim}(v)+1 .
$$

ДоказательСтво. Индукция по коразмерности $s=d-\operatorname{dim}(v)$. База индукции для $s=$ $0,1,2$. Предположение индукции: неравенство выполняется для всех плоскостей всех наборов с коразмерностью не более $s-1$. Переход индукции: выберем из набора $s=d-\operatorname{dim}(v)$ гиперплоскостей $p_{1}, \ldots, p_{s}$, пересечение которых является плоскостью $v$. Остальные гиперплоскости набора обозначим через $p_{s+1}, \ldots, p_{n}$. Рассмотрим последовательность наборов гиперплоскостей $\mathscr{A}_{0}, \ldots, \mathscr{A}_{n-s}$, где

$$
\mathscr{A}_{0}=\left\{p_{1}, \ldots, p_{s}\right\} \quad \text { и } \quad \mathscr{A}_{i}=\mathscr{A}_{i-1} \cup p_{s+i} .
$$

Заметим, что $\mu_{\mathscr{A}_{0}}(v)=(-1)^{s}$. По лемме 3 имеем

$$
\mu_{\mathscr{A}_{i}}(v)= \begin{cases}\mu_{\mathscr{A}_{i-1}}(v), & \text { если } v \nsubseteq p_{s+i}, \\ \mu_{\mathscr{A}_{i-1}}(v)-\mu_{p_{s+i} \cap \mathscr{A}_{i-1}}(v), & \text { если } v \subseteq p_{s+i} .\end{cases}
$$

Заметим, что

$$
\operatorname{sign} \mu_{\mathscr{A}_{i-1}}(v)=(-1)^{d+\operatorname{dim}(v)} \quad \text { и } \quad \operatorname{sign} \mu_{p_{s+i} \cap \mathscr{A}_{i-1}}(v)=(-1)^{d-1+\operatorname{dim}(v)},
$$

так как $\operatorname{sign} \mu(u, v)=(-1)^{\operatorname{dim} u+\operatorname{dim} v}$ для любой пары плоскостей $u \preceq v$. Поэтому в случае $v \subseteq p_{s+i}$

$$
\left|\mu_{\mathscr{A}_{i}}(v)\right| \geqslant\left|\mu_{\mathscr{A}_{i-1}}(v)\right|+1 .
$$

Лемма 7. Для нетривиального набора $\mathscr{A}$ гиперплоскостей в пространстве $\mathbb{R P}^{d}$ и любого иелого $1 \leqslant i \leqslant d$

$$
\sum_{\operatorname{dim}(v)=d-i}|\mu(v)| \geqslant(m-d+1) \frac{C_{n}^{i}}{C_{m-d+i}^{i}} .
$$

ДокАзАТЕльство. Обозначим через $a_{j}^{(d-i)}$ число $(d-i)$-мерных плоскостей из множества $L(\mathscr{A})$, принадлежащих $j$ гиперплоскостям набора $\mathscr{A}$, где $i \leqslant j \leqslant m-(d-i)$ (заметим, что $(d-i)$-мерная плоскость не может принадлежать более чем $m-(d-i)$ гиперплоскостям, так как найдутся еще не менее чем $d-i$ гиперплоскостей, с которыми у нее непустое пересечение). Рассмотрим пары: поднабор из $i$ гиперплоскостей $p_{k_{1}}, \ldots, p_{k_{i}}$ набора $\mathscr{A}$ 
и плоскость $v \in L(\mathscr{A})$ размерности $d-i$, принадлежащая всем гиперплоскостям $p_{k_{1}}, \ldots, p_{k_{i}}$. Число таких пар не меньше числа поднаборов, т.е. не меньше $C_{n}^{i}$. При этом плоскость, принадлежащая $j$ гиперплоскостям, будет учтена $C_{j}^{i}$ раз. Поэтому

$$
C_{n}^{i} \leqslant \sum_{j=i}^{m-(d-i)} a_{j}^{(d-i)} C_{j}^{i} \leqslant \sum_{j=i}^{m-(d-i)} a_{j}^{(d-i)}(j-i+1) \frac{C_{m-d+i}^{i}}{m-d+1} .
$$

По лемме 6 для плоскости $v \in L(\mathscr{A})$ размерности $d-i$ имеем $|\mu(v)| \geqslant(j-i+1)$, поэтому

$$
\sum_{j=i}^{m-(d-i)} a_{j}^{(d-i)}(j-i+1) \leqslant \sum_{\operatorname{dim}(v)=d-i}|\mu(v)| .
$$

СледствиЕ 1 (из лемм 4 и 7). Имеем (берем $j=(d-i) / 2)$ :

$$
f \geqslant(m-d+1) \sum_{j=0}^{[(d-1) / 2]} \frac{C_{n}^{d-2 j}}{C_{m-2 j}^{d-2 j}} \geqslant(m-d+1) \frac{C_{n}^{d}}{C_{m}^{d}} .
$$

ДокАЗАТЕЛЬСТво тЕОРЕмы 1. Построим примеры (а)-(г) наборов гиперплоскостей с указанными числами $f$ областей, указав системы точек $A_{1}, \ldots, A_{n}$, проективно двойственных гиперплоскостям.

(a) Пусть точки $A_{1}, \ldots, A_{n-d+1}$ лежат на одной прямой, точки $A_{n-d+2}, \ldots, A_{n}$ находятся в общем положении по отношению друг к другу и к предыдущим точкам. Тогда проективно двойственный набор гиперплоскостей будет минимальным и $f=(n-d+1) 2^{d-1}$.

(б) Пусть точки $A_{1}, \ldots, A_{n-d}$ лежат на одной прямой, точки $A_{1}, A_{n-1}, A_{n}$ лежат на одной прямой, и других вырождений между точками $A_{1}, \ldots, A_{n}$ нет. Тогда $f=3(n-d) 2^{d-2}$.

(в) Пусть точки $A_{1}, \ldots, A_{n-d}$ лежат на одной прямой, точки $A_{1}, A_{2}, A_{n-1}, A_{n}$ лежат в одной трехмерной плоскости, и других вырождений между точками $A_{1}, \ldots, A_{n}$ нет. Тогда $f=(3 n-3 d+1) 2^{d-2}$.

(г) Пусть точки $A_{1}, \ldots, A_{n-d}$ лежат на одной прямой, точки $A_{1}, A_{n-2}, A_{n-1}, A_{n}$ лежат в одной трехмерной плоскости, и других вырождений между точками $A_{1}, \ldots, A_{n}$ нет. Тогда $f=7(n-d) 2^{d-3}$.

Докажем по индукции по $d$, что остальные реализуемые числа областей (для данных чисел $n$ и $d$ ) больше, чем $7(n-d) 2^{d-3}$. Если $m=d$, то гиперплоскости набора находятся в общем положении и число областей максимальное возможное. Для $m=d+1$ по следствию 1 имеем

$$
f \geqslant 2 \frac{C_{n}^{d+1}}{n-d} \geqslant 7(n-d) 2^{d-3}
$$

при $n \geqslant 2 d+5$. Далее и в базе, и в переходе индукции будем считать, что $m \geqslant d+2$.

База индукции: $d=3, n \geqslant 11$. Пересекающиеся в одной точке $m$ плоскостей образуют конус над двумерной конфигурацией прямых, поэтому число областей, образуемых ими без оставшихся плоскостей, равно числу областей $\varphi \in F_{2}(m)$ двумерной конфигурации, и известно по работе Мартинова [2]. Возможны три случая.

Случай 1) $m=n-1$. Тогда $f(\mathscr{A})=2 \varphi$, где $\varphi \in\{2 n-4,3 n-9,3 n-8\}$ или $\varphi \geqslant 4 n-16$.

Случай 2) $5 \leqslant m \leqslant n-3$. По лемме 5 имеем $f \geqslant 2(n-m+1)(m-1) \geqslant 8 n-32 \geqslant 7 n-21$ при $n \geqslant 11$.

Случай 3) $m=n-2$. Обозначим через $l$ прямую пересечения двух оставшихся плоскостей; если прямая $l$ принадлежит объединению $m$ плоскостей, то $f=3 \varphi$, где

$$
\varphi=2 n-6 \quad \text { или } \quad \varphi \geqslant 3 n-12 .
$$

Если прямая $l$ не принадлежит объединению $m$ плоскостей, то $f=3 \varphi+b$, где $b$ равно числу точек пересечения $m$ плоскостей с прямой $l$. Если $\varphi \geqslant 3 n-12$, то, очевидно, $f \geqslant 7 n-21$. 
Если $\varphi=2 n-6$, то $m$ плоскостей образуют конус над минимальной конфигурацией $n-2$ прямых в $\mathbb{R P}^{2}$ и поэтому из $m$ плоскостей все, кроме одной, проходят через проективную прямую, не пересекающуюся с прямой $l$. Значит, точки пересечения прямой $l$ с этими $n-3$ плоскостями различны и $b \geqslant n-3$. Откуда $f \geqslant 7 n-21$. База индукции проверена.

Предположение индукции - первые 4 числа множества $F_{p}(n)$ имеют указанный вид для любых целых чисел $3 \leqslant p<d$ и $n \geqslant 2 p+5$. Переход индукции: возьмем нетривиальный набор $\mathscr{A}$ гиперплоскостей в пространстве $\mathbb{R} \mathbb{P}^{d}$. Ввиду первого после примера абзаца доказательства можно считать $m \geqslant d+2$; рассмотрим три случая.

Случай 1) $m=n-1$. Тогда $f(\mathscr{A})=2 \varphi$, где $\varphi \in F_{d-1}(n-1)$. Применяя предположение индукции к множеству $F_{d-1}(n-1)$, получим, что либо $\varphi$ совпадает с одним из трех чисел

$$
(n-d+1) 2^{d-2}, \quad 3(n-d) 2^{d-3}, \quad(3 n-3 d+1) 2^{d-3},
$$

либо $\varphi \geqslant 7(n-d) 2^{d-4}$.

Случай 2) $d+2 \leqslant m \leqslant n-3$. По лемме 5 имеем

$$
f \geqslant(n-m+1)(m-d+2) 2^{d-2} \geqslant(4 n-4 d-4) 2^{d-2} \geqslant 7(n-d) 2^{d-3}
$$

при $n \geqslant d+8$.

Случай 3) $m=n-2$. Рассмотрим $n-2$ гиперплоскости $p_{1}, \ldots, p_{n-2}$, имеющих общую точку. Они делят пространство $\mathbb{R P}^{d}$ на $\varphi$ областей, где $\varphi \in F_{d-1}(n-2)$. Обозначим через $l$ пересечение двух оставшихся гиперплоскостей $p_{n-1}$ и $p_{n}$. По предположению индукции

$$
\varphi=(n-d) 2^{d-2} \quad \text { или } \quad \varphi \geqslant 3(n-d-1) 2^{d-3}
$$

(предположение индукции можно использовать, так как $n-2 \geqslant 2(d-1)+5)$. Если $l \in$ $\bigcup_{i=1}^{n-2} p_{i}$, то $f=3 \varphi$ и случай $m=n-2$ разобран. Если $l \notin \bigcup_{i=1}^{n-2} p_{i}$, то обозначим через $\mathscr{B}$ множество плоскостей $p_{i} \cap l$ в плоскости $l$, где $l$ рассматривается как объемлющее $(d-2)$-мерное проективное пространство. По лемме 2 число областей в конфигурации $\mathscr{A}$ равно $3 \varphi+f(\mathscr{B})$, и, если $\varphi \geqslant 3(n-d-1) 2^{d-3}$, то случай $m=n-2$ разобран. Если $\varphi=$ $(n-d) 2^{d-2}$, то по лемме 1 плоскости $p_{1}, \ldots, p_{n-2}$ образуют минимальный набор, в котором гиперплоскости $p_{1}, \ldots, p_{n-d}$ проходят через общую $(d-2)$-мерную плоскость $l_{0}$, а каждая из гиперплоскостей $p_{n-d+1}, \ldots, p_{n-2}$ находится в общем положении по отношению к остальным (кроме себя) гиперплоскостям $p_{1}, \ldots, p_{n-2}$. Докажем, что тогда $\mathscr{B}$ - нетривиальный набор как минимум $n-3$ плоскостей в пространстве $l$; нетривиальность очевидна. Заметим, что размерность пересечения $l_{0} \cap l$ равна $d-4$, так как иначе по соображениям размерности в наборе $\mathscr{A}$ нашлась бы $n-1$ гиперплоскость с не пустым пересечением, а именно, все, кроме одной из гиперплоскостей $p_{n-d+1}, \ldots, p_{n-2}$, что противоречит равенству $m=$ $n-2$. Плоскость $l$ пересекается с каждой из гиперплоскостей $p_{1}, \ldots, p_{n-2}$ по $(d-3)$-мерной плоскости из набора $\mathscr{B}$. Если в наборе $\mathscr{B}$ меньше чем $n-3$ плоскостей, то выполняется один из двух вариантов:

- $\operatorname{dim} p_{i} \cap p_{j} \cap p_{k} \cap l=d-3$ для некоторых индексов $i<j<k \leqslant n-2$,

- $\operatorname{dim} p_{i} \cap p_{j} \cap l=\operatorname{dim} p_{k} \cap p_{r} \cap l=d-3$ для различных индексов $i, j, k, r$, не превосходящих числа $n-2$.

Рассмотрим первый случай. Из трех индексов $i, j$ и $k$ хотя бы два больше, чем $n-d$ (так как иначе $\operatorname{dim} l_{0} \cap l \geqslant d-3$ ). Если $d=4$, то это будут индексы $j=n-3$ и $k=$ $n-2$. Тогда прямая $p_{i} \cap p_{n-3} \cap p_{n-2}$ содержит точку пересечения $n-2$ гиперплоскостей, но одновременно принадлежит плоскости $l$, значит, все $n$ гиперплоскостей набора $\mathscr{A}$ проходят через одну точку - противоречие с условием $m=n-2$. Если $d \geqslant 5$, то гиперплоскости (из них не менее $n-d+4$ различных)

$$
p_{1}, \ldots, p_{n-d}, p_{i}, p_{j}, p_{k}, p_{n-1}, p_{n}
$$


пересекаются по плоскости размерности не менее чем $d-5$, поэтому вместе с еще $(d-5)$-ю гиперплоскостями набора $\mathscr{A}$ имеют общую точку, что противоречит условию $m=n-2$.

Рассмотрим второй случай. Заметим, что

$$
\operatorname{dim} p_{i} \cap p_{j} \cap p_{k} \cap p_{r} \cap p_{n-1} \cap p_{n} \geqslant d-4 .
$$

Не более двух индексов из $i, j, k$ и $r$ не превосходят $n-d$. Возможны три случая: таких индексов два, один или нет.

(а) Если таких индексов два, то можно считать, что $i<k \leqslant n-d$. Тогда

$$
l \cap l_{0}=l_{0} \cap p_{j} \cap p_{r}
$$

и все $n$ гиперплоскостей набора $\mathscr{A}$ имеют общую точку.

(б) Если такой индекс один, то можно считать, что $i \leqslant n-d$. Тогда $d \geqslant 5$ и размерность пересечения гиперплоскостей

$$
p_{1}, \ldots, p_{n-d}, p_{k}, p_{r}, p_{n-1}, p_{n}
$$

не менее, чем $d-5$. Поэтому они вместе с еще $(d-5)$-ю гиперплоскостями набора $\mathscr{A}$ (выбранных из гиперплоскостей $p_{n-d+1}, \ldots, p_{n-2}$ ) имеют общую точку.

(в) Если таких индексов нет, то $d \geqslant 6$ и размерность пересечения плоскостей

$$
p_{1}, \ldots, p_{n-d}, p_{i}, p_{j}, p_{k}, p_{r}, p_{n-1}, p_{n}
$$

не меньше, чем $d-6$. Поэтому все гиперплоскости набора $\mathscr{A}$ имеют не пустое пересечение. Во всех трех подслучаях (а)-(в) получили противоречие с условием $m=n-2$.

Вернемся к оценке числа $f$. Раз $\mathscr{B}$ - нетривиальный набор из не менее, чем $n-3$ плоскостей, то по лемме 1 имеем $f(\mathscr{B}) \geqslant(n-d) 2^{d-3}$ и

$$
f(\mathscr{A})=3 \varphi+f(\mathscr{B}) \geqslant 7(n-d) 2^{d-3} .
$$

\section{СПИСОК ЦИТИРОВАННОЙ ЛИТЕРАТУРЫ}

[1] B. Grünbaum, Arrangements and Spreads, Amer. Math. Soc., Providence, RI, 1972. [2] N. Martinov, Discrete Comput. Geom., 9:1 (1993), 39-46. [3] R. W. Shannon, J. Combinatorial Theory Ser. A, 20:3 (1976), 327-335. [4] T. Zaslavsky, Mem. Amer. Math. Soc., 1, no. 154, Amer. Math. Soc., Providence, RI, 1975. [5] R. C. Buck, Amer. Math. Monthly, 50 (1943), 541-544. [6] P. Orlic, L. Solomon, Invent. Math., 56:2 (1980), 167-189. [7] P. Deshpande, Arrangements of Submanifolds and the Tangent Bundle Complement, Electronic Thesis and Dissertation Repository, Paper 154, 2011. [8] И. Н. Шнурников, "Конфигурации подмногообразий коразмерности 1", Матем. сб., 203:9 (2012), 133-160.

И. Н. Шнурников

Национальный исследовательский университет

«Высшая школа экономики», г. Москва

E-mail: shnurnikov@yandex.ru
Поступило

27.07.2014 